\title{
Vasculitis reumatoide: a propósito de dos casos
}

\section{(Rheumatoid vasculitis: about two cases)}

Marvin Sánchez-Benavides

\section{Resumen}

La vasculitis reumatoide constituye una complicación inusual, pero severa, de la artritis reumatoide, caracterizada por un proceso inflamatorio que compromete vasos sanguíneos de pequeño y mediano calibre, asociada o no a manifestaciones extraarticulares.

Algunos factores predisponentes descritos incluyen ciertos haplotipos de antígenos leucocitarios humanos, fumado y enfermedad de larga data. Una disminución en la incidencia ha sido notada desde los años 90 y confirmada en estudios recientes, asociada en particular a un diagnóstico precoz de artritis reumatoide y estrategias terapéuticas que incluyen un aumento en la prescripción de metotrexate. La vasculitis reumatoide se asocia a tasas de mortalidad hasta del 40\% a los 5 años y morbilidad importante. Se presentan dos pacientes con diagnóstico previo de artritis reumatoide, con un cuadro crónico de úlceras y disestesias en miembros inferiores, asociado a síndrome anémico. Los estudios histológicos revelaron vasculitis de mediano vaso compatible con vasculitis reumatoide. Se inició manejo con inmunosupresores más esteroides, con resolución del cuadro clínico inicial.

Descriptores: vasculitis reumatoide, artritis reumatoide, manifestaciones extraarticulares.

\section{Abstract}

Rheumatoid vasculitis remains a rare but serious complication of rheumatoid arthritis characterized by an inflammatory process that primarily affects small to medium-sized blood vessels, it can be associated with other extra-articular manifestations. A number of predictor or predisposing factors including certain human leukocyte antigen haplotypes, smoking and long-standing disease. A declining trend in the incidence of rheumatoid vasculitis

Trabajo realizado y afiliación del autor: Servicio de Reumatología del Hospital "Dr. Rafael Ángel Calderón Guardia"

Conflicto de interés: no existen conflicto de interéses

Fuentes de apoyo: No aplica.

凶marsbcrg@mail.com

ISSN 0001-6012/2018/60/2/34-37

Acta Médica Costarricense, () 2018

Colegio de Médicos y Cirujanos

de Costa Rica has been noted since the 1990s and reconfirmed in recent studies, related to early diagnosis of rheumatoid arthritis, and widespread use of methotrexate. Rheumatoid vasculitis is associated with high rates of premature mortality with up to $40 \%$ of patients dying by 5 years, as well as a significant morbidity. We present two patients with diagnosis of rheumatoid arthritis with a chronic condition of ulcers and dysesthesias on legs associated with anemic syndrome; the histological study revealed medium size vasculitis. Treatment with inmunosuppresants and steroids was given with resolution of symptoms.

Keywords: rheumatoid vasculitis; rheumatoid arthritis; extra-articular manifestations.

Fecha recibido: 24 de enero 2018

Fecha aprobado: 27 de febrero 2018

La vasculitis reumatoide (VR) constituye una complicación infrecuente y severa de la artritis reumatoide (AR), caracterizada por un proceso inflamatorio que afecta vasos sanguíneos pequeños y medianos, clínicamente heterogénea, asociada o no a otras manifestaciones extraarticulares de AR. ${ }^{1,2}$

Algunos factores predisponentes descritos para VR incluyen ciertos haplotipos de antígenos leucocitarios humanos, sexo masculino, fumado y enfermedad erosiva de larga data. . $^{1,3,4}$ Una disminución en la incidencia ha sido observada desde los años 90, asociada especialmente a un diagnóstico precoz de AR y estrategias terapéuticas que contemplan un aumento en la prescripción de metotrexate. . $^{1,2,4}$ Así mismo, VR se asocia a altas tasas de morbimortalidad. ${ }^{1,6,7}$ A la fecha, no existe bibliografía relacionada con VR en nuestro país; se reportan 2 pacientes con dicho diagnóstico del Hospital "Dr. Rafael Ángel Calderón Guardia”.

\section{Caso clínico 1}

Femenina de 57 años, con diagnóstico de AR en 2005, sin control desde 2009, referida por un cuadro de tres meses de evolución de síntomas constitucionales, asociado a lesiones ulceradas y dolor quemante en miembros inferiores. La evaluación física documenta secuelas de AR en articulaciones de ambas manos, así como lesiones ulceradas con borde activo y necrosis periférica, e hipoestesias en miembros inferiores. Los estudios de laboratorio reportaron un síndrome anémico y elevación en los reactantes de fases aguda. El examen general de orina presentó proteinuria en $500 \mathrm{mg} / \mathrm{dL}$ y la orina de $24 \mathrm{~h}$ reportó proteinuria de 7,2g/24horas. El factor reumatoide se reportó en $208 \mathrm{UI} / \mathrm{ml}$ y el antipéptido cíclico citrulinado mayor a 200U/ml. Los anticuerpos antinucleares, antiADN doble cadena, complemento C3 y C4, ANCAc y ANCAp, se reportaron negativos. La velocidad de conducción nerviosa documentó una 
polineuropatía axonal sensitiva-motora en miembros inferiores. La biopsia renal reportó una glomerulonefritis membranosa y la de piel, un infiltrado linfocitario perivascular y perineural con compromiso en la pared vascular.

Ante la sospecha clínica de VR se inician esteroides intravenosos por 3 días, seguidos de dosis descendente de esteroides orales, Azatioprina $100 \mathrm{mg}$ /día, más terapia antirresortiva (calcio $600 \mathrm{mg} /$ día, vitamina D 2 gotas/día y alendronato $70 \mathrm{mg}$ /semana). En Consulta Externa se valoró paciente sin lesiones cutáneas, disestesias o parestesias; los laboratorios reportaron resolución del síndrome anémico y normalización en los reactantes de fases aguda. Orina de 24 horas reportó proteinuria de $231 \mathrm{mg} / 24$ horas.

\section{Caso clínico 2}

Femenina de 25 años, con antecedente de hipertensión arterial y AR diagnosticada y manejada en primer nivel de atención desde 2010, con prednisona oral a dosis de 20$30 \mathrm{mg} /$ día, referida para iniciar control en Consulta Externa con historia de un año de evolución de lesiones ulceradas en miembros inferiores, asociado a disestesias y parestesias. El examen físico evidenció un aspecto cushingoide franco, secuelas de AR con una franca poliartritis en rodillas, codos y pequeñas articulaciones de manos, asociado a lesiones ulceradas en miembros inferiores, además de hipoestesia en manos y pies. Los estudios de laboratorio reportaron síndrome anémico, trombocitos y elevación de los reactantes de fase aguda. Los exámenes generales de orina y orina de 24 horas se reportaron en rangos normales. El factor reumatoide se reportó en $2206 \mathrm{UI} / \mathrm{ml}$ y antipéptido cíclico citrulinado en $70.7 \mathrm{U} / \mathrm{ml}$. Los anticuerpos antinucleares, niveles de complemento C3 y
C4, antiADN doble cadena, ANCAc y ANCAp negativos. La velocidad de conducción nerviosa fue reportada normal. La biopsia cutánea fue compatible con vasculitis de mediano vaso.

Ante sospecha de VR, se inicia terapia con metotrexate 8 comprimidos/semana, leflunomida $20 \mathrm{mg} /$ día, terapia antirresortiva y prednisona $10 \mathrm{mg} \mathrm{c} / 8$ horas, en dosis descendientes. En la Consulta Externa se valoró sin actividad articular inflamatoria o lesiones en piel; los laboratorios reportaron franca mejoría del síndrome anémico, sin trombocitos y disminución significativa en reactantes de fase aguda. El examen general de orina y la bioquímica no reportaron hallazgos patológicos.

\section{Discusión}

La VR fue descrita inicialmente a finales del siglo XIX en un paciente con AR e historia de neuritis, en quien se documentó un infiltrado inflamatorio en la vasa nervorum, ${ }^{1,3,5}$ dicha entidad presenta una amplia gama de manifestaciones clínicas de acuerdo con el órgano comprometido., ${ }^{5,8}$

Desde el punto de vista epidemiológico, se ha observado una disminución en la incidencia de VR desde la década de 1990. Una revisión de manifestaciones extraarticulares en AR reportó una disminución en la incidencia acumulada de VR del 3,6\% en 1985-1994 al 0,6\% durante 1995-2007.5 Esta menor incidencia ha sido atribuida a factores como disminución en las tasas de tabaquismo activo, introducción de la terapia biológica y cambios en la prescripción de fármacos modificadores de enfermedad reumática, especialmente por incrementos en el

\begin{tabular}{|c|c|c|}
\hline \multicolumn{3}{|c|}{ Cuadro 1. Manifestaciones clínicas frecuentes en vasculitis reumatoide } \\
\hline $\begin{array}{l}\text { Órgano } \\
\text { clínica }\end{array}$ & Manifestación & $\begin{array}{l}\text { Frecuencia } \\
\qquad(\%)\end{array}$ \\
\hline Piel & $\begin{array}{l}\text { Petequias, púrpura, úlceras } \\
\text { Lesiones isquémicas digitales } \\
\text { Pioderma gangrenoso } \\
\text { Eritema elevatum diutinium }\end{array}$ & 90 \\
\hline Manifestaciones sistémicas & Fiebre, pérdida de peso & 80 \\
\hline Nervios periféricos & Mononeuritis múltiple & 40 \\
\hline Corazón & $\begin{array}{c}\text { Pericarditis, arteritis coronaria } \\
\text { Arritmias }\end{array}$ & 30 \\
\hline Compromiso ocular & Keratitis ulcerativa & 30 \\
\hline Tracto gastrointestinal & Isquemia intestinal & Raro \\
\hline Pulmones & $\begin{array}{c}\text { Hemorragia alveolar difusa } \\
\text { Pleuritis }\end{array}$ & Raro \\
\hline Riñones & Glomerulonefritis Amiloidosis & Raro \\
\hline Sistema nervioso central & $\begin{array}{l}\text { Hemiparesia, convulsiones } \\
\text { Estado confusional }\end{array}$ & Raro \\
\hline
\end{tabular}


uso de metotrexate. ${ }^{1,3,5}$ A pesar de la menor incidencia, las tasas de mortalidad se mantienen constantes, incluyendo tasas de mortalidad al año del $12 \%$ y a 5 años, del $40 \%$ al $60 \%$.,5,6,8 En nuestro país se desconoce la incidencia a la fecha, dada la ausencia de estudios epidemiológicos; no existe, al menos en la bibliografía nacional, reportes de casos de VR.

La patogenia de VR no ha sido del todo dilucidada; clásicamente el compromiso se centra en vasos de pequeño y mediano calibre similar a poliarteritis nodosa, aunque a diferencia de esta, no se asocia a formación de aneurismas vasculares. ${ }^{1}$ Algunos factores genéticos como el HLA-DRB1, se han asociado a manifestaciones extraarticulares en AR, incluyendo VR, particularmente el HLA-DRB1 *0401/*0401, *0401/*0404 y *0101/*0401.,

Los complejos inmunes desempeñan un papel primordial en la patogenia, así como ciertos anticuerpos contra células endoteliales, la glucosa 6-fosfoisomerasa y la fracción C1q del complemento. El depósito de complejos inmunes en tejidos induce cambios inflamatorios locales por activación del complemento y la unión con la fracción cristalizable de células de superficie, provocando degranulación, fagocitosis, expresión de moléculas de adhesión y liberación de citoquinas como el factor de necrosis tumoral alfa (TNF-alfa), interleuquina 1 (IL-1) e interleuquina 6 (IL-6).,4,9

Algunos factores clínicos como edad temprana de inicio de AR, el factor reumatoide, sexo masculino, enfermedad de larga data agresiva con presencia de erosiones radiológicas y tabaquismo activo, han sido descritos como importantes en la patogenia de VR. ${ }^{1,4-6,10}$ El tabaquismo se ha asociado a efectos proinflamatorios endoteliales intimales y disfunción endotelial con vasculopatía oclusiva. ${ }^{1}$ Nuestras pacientes descritas presentaron una enfermedad erosiva, sin control reumatológico, con seropositivad para el factor reumatoide y antipepito cíclico citrulinado, una de las cuales fue diagnosticada a los 20 años de edad; en ningún caso se describió tabaquismo activo previo.

El promedio entre el inicio de la AR y el desarrollo de VR es de 10 a 14 años. ${ }^{1-3,6}$ En este caso, el cuadro clínico debutó más tempranamente respecto a lo descrito en la bibliografía, y es probable que esto se relacione con la falta de prescripción de fármacos modificadores de enfermedad reumática, en ambos casos.

Usualmente, el cuadro clínico se acompaña de síntomas constitucionales como sensación febril; ;,4,6 los hallazgos son heterogéneos y dependerán del tamaño del vaso y órgano comprometido, afectando uno o varios de manera simultánea. ${ }^{1,4-6,9}$ El compromiso en piel y sistema nervioso periférico es lo que con mayor frecuencia se describe en más del $80 \%$ de los casos, similar a lo observado en nuestras pacientes, seguido de la afección ocular y pericárdica. ${ }^{1,3,6}$ Se ha reportado compromiso del tracto gastrointestinal en forma de lesiones isquémicas o perforación intestinal, así como afección renal, cardiaca y en sistema nervioso central, en porcentajes menores. ${ }^{1,2,11}$ Fue llamativo el compromiso renal documentado en una paciente como glomerulonefritis membranosa con proteinuria en rango nefrótico, la cual resolvió con la instauración de inmunosupresión farmacológica.

Algunos hallazgos de laboratorio incluyen anemia de enfermedad crónica, trombocitosis, elevación en reactantes de fase aguda, hallazgos similares a los descritos en las pacientes. Ocasionalmente, se observa hipocomplementemia, crioglobulinemia, e incluso se ha demostrado en algunos pacientes la presencia de anticuerpos anticitoplasma de neutrofilo (ANCA), ${ }^{1,5,6}$ sobre todo dirigidos contra lactoferrina. ${ }^{1}$

El diagnóstico definitivo requiere evidencia histológica de vasculitis del órgano afectado. Con frecuencia se realiza biopsia de piel o nervio periférico. ${ }^{1,4,6}$ La angiografía se reserva a casos con sospecha de compromiso mesentérico. ${ }^{1}$ El estudio histológico demuestra presencia de infiltrado mononuclear o neutrofílico en las paredes de los vasos afectados, asociado a necrosis, leucocitoclastia y disrupción de la lámina elástica, 4,5 hallazgos descritos en el estudio histológico de las pacientes.

La ausencia de criterios de clasificación de VR y la heterogeneidad clínica inciden en un abordaje terapéutico primordialmente empírico, basado en reportes de casos o series pequeñas. ${ }^{1,6,10}$ La agresividad del tratamiento dependerá del órgano comprometido; para el compromiso cutáneo y neuropático, algunos autores recomiendan metotrexate $10 \mathrm{mg}$ $25 \mathrm{mg}$ semanales, o azatioprina a $2 \mathrm{mg} / \mathrm{kg}$ peso, con porcentajes altos de remisión; ${ }^{6}$ dicho abordaje terapéutico fue la elección en nuestros pacientes con resolución de las manifestaciones clínicas iniciales. El compromiso severo suele requerir manejo con altas dosis de esteroides más ciclofosfamida o agentes biológicos; ;,4 no obstante, algunos reportes han relacionado el uso de ciclofosfamida como predictor de recaídas, daño a órgano blanco y procesos infecciosos severos asociados. ${ }^{1,6}$

Hay reportes de tratamiento exitoso con terapia anti TNFalfa, antilL-6, antilL-1, antiCD20 y abatacept. ${ }^{1,4,6,10}$ Así mismo, se ha descrito el desarrollo de VR asociado a terapia antiTNFalfa en relación con reacciones de hipersensibilidad tipo III, entre otros factores. ${ }^{1}$

A pesar de la disminución en la incidencia y el incremento en el uso de citotóxicos y la terapia biológica, la severidad y mortalidad a 5 años ha permanecido sin cambios desde los años 80. Se han reportado tasas de mortalidad que varían desde el $26 \%$ hasta el $60 \%$ en diversos estudios epidemiológicos. ${ }^{1}$

La VR es una entidad clínica inusual, especialmente por un mejor control clínico de AR. A pesar de la disminución en la incidencia en los últimos años, aún constituye una causa importante de morbimortalidad precoz en pacientes con AR.

\section{Referencias}

1. Makol A, Matteson EL, Warrington KJ. Rheumatoid vasculitis: an update. Curr Opin Rheumatol 2015,27:63-70

2. Gorman DJ, David-Vaudey E, Pai M, Lum RF, Criswell LA. Particular HLADRB1 shared epitope genotypes are strongly associated with rheumatoid vasculitis. Arthritis Rheum 2004;50:3476-3484 


\section{Vasculitis reumatoide / Sánchez- Benavides}

3. Watts RA, Mooney J, Lane SE, Scott DGI. Rheumatoid vasculitis: becoming extint. Rheumatology (Oxford) 2004;43:920-923

4. Genta MS, Genta RM, Gabay C. Systemic Rheumatoid Vasculitis: A review. Semin Arthritis Rheum 36:88-98,2006

5. Ntatsaki EM, Mooney J, Scott DGI, Watts RA. Systemic rheumatoid vasculitis in the era of modern immunosuppressive therapy. Rheumatology 2014;53:145152

6. Makol A, Crowson CS, Wetter DA, Sokumbi O, Matteson EL, Warrington KJ. Vasculitis associated with rheumatoid arthritis: a cae-control study. Rheumatology (Oxford) 2014;53:890-899

7. Matsunawa M, Isozaki T, Osai T, Yajima N, Takeuchi HT, Negishi M et al. Increased serum levels of soluble fractalkine (CX3CL1) correlated with disease activity in rheumatoid vasculitis. Arthritis Rheum. 2006;54:3408-3416
8. Tago M, Naito Y, Aihara H, Furkawa N, Yamashita S. Recurrent stenosis of the ileum caused by rheumatoid vasculitis. Intern Med. 2016;55(7):819-823

9. Voskuyl AE, Hazes JmW, Zwinderman AH, Paleolog EM, van deer Meer FJM et al. Diagnostic strategy for the assessment of rheumatoid vasculitis. Ann Rheum Dis 2003;62:407-413

10. Watts, RA. Rheumatoid vasculitis-down but not out. Nat. Rev. Rheumatol. 10,281-282 (2014).

11. Parker B, Chattopadhyay C. A case of rheumatoid vasculitis involving the gastrointestinal tract in early disease. Rheumatology (Oxford) 2007;46:1737. 1738

\section{Instrucciones para autores}

Acta Médica Costarricense (AMC) es la publicación científica oficial del Colegio de Médicos y Cirujanos de Costa Rica. La revista publica trabajos inéditos que pueden ser originales, de revisión, de opinión, comunicaciones breves, cartas al editor y editoriales. Artículos relacionados con las ciencias biomédicas básicas, son considerados en el tanto que ilustren su implicación con la clínica. En todos los casos, lo que se escriba debe dirigirse a la audiencia médica general.

Los manuscritos presentados a consideración del Comité Editorial (CE), para publicarse en AMC, deben estructurarse de acuerdo con los "Requisitos uniformes para manuscritos enviados a revistas biomédicas", del Comité Internacional de Editores de Revistas Médicas, cuya última versión puede accederse en http://www.icmje.org. Los manuscritos enviados en forma inadecuada serán devueltos al autor.

Los artículos aceptados para publicación en AMC pasan a ser propiedad del Colegio de Médicos y Cirujanos de Costa Rica y no deben haber sido publicados total o parcialmente en ninguna otra revista o medio electrónico, aunque sea en otro idioma.

\section{Instrucciones generales}

El manuscrito se ordena de la siguiente manera: página de título, resumen en español e inglés, texto principal, agradecimientos y colaboradores, referencias, cuadros, leyendas de las figuras y figuras.

\section{Página de título}

Título. En español e inglés, conciso, pero informativo en cuanto al objetivo del trabajo, sólo la primera letra de la primera palabra debe venir en mayúscula. Debe incluir la información necesaria para permitir una recuperación electrónica eficiente. No debe contener abreviaturas. En caso de estudios en animales, se debe mencionar la especie.

Autores. Primer nombre y 2 apellidos de todos los autores (insertar guion entre el primer y segundo apellidos) y su afiliación institucional (lugar de trabajo actual). Deben listarse como autores quienes contribuyeron sustancialmente en el diseño del estudio, adquisición o análisis de la información, redacción del manuscrito o revisión crítica y aprobación de la versión final. Es preciso especificar la contribución de cada autor al trabajo según estas áreas señaladas.

Nombre del departamento e institución donde se realizó el trabajo.

Descriptores. De 4-5 palabras claves en español e inglés (keywords), estos son términos que orientan sobre el tema de la publicación y permiten su búsqueda en los medios digitales.

\section{Título corto. Máximo 45 caracteres}

Abreviaturas: todas las que se utilizan en el texto, listadas en orden alfabético. Solo se abrevian los términos empleados en más de 3 ocasiones y la abreviatura se debe definir luego de la primera mención en el texto. No se deben usar abreviaturas en el resumen.
Listar número de páginas, figuras y cuadros.

Correspondencia. Correo electrónico y teléfonos del autor a quien se debe dirigir la correspondencia.

Fuentes de apoyo. Listado de quienes contribuyeron económicamente, con equipo, medicamentos u otros.

Conflictos de interés. Declaración detallada de todos los potenciales conflictos de interés de los autores.

\section{Tipos de artículos y sus requerimientos}

\section{Artículo original}

Resumen. En español e inglés, máximo 500 palabras, estructurado, utilizando los siguientes subtítulos: justificación, métodos, resultados y conclusión/es (background, methods, results and conclusion/s). No deben usarse abreviaturas, referencias o notas al pie de página.

Introducción. Expresa el propósito del artículo, plantea el objetivo en forma clara y precisa (justifica la necesidad e importancia de la investigación). Ubica el tema dentro del conocimiento actual, evitando una revisión muy extensa de la bibliografía o un resumen de los resultados.

Métodos. Se describe con claridad el diseño del estudio, método de selección de participantes (criterios de inclusión y exclusión), instrumentos, análisis de laboratorio, procedimientos u otros utilizados con suficiente detalle como para que sean reproducibles. Manuscritos que reporten información obtenida en seres humanos deben incluir una nota en la sección de métodos, que asegure que el protocolo del estudio cumplió con los requerimientos del Comité Ético-Científico (CEC) del centro correspondiente, o en su defecto de un CEC aprobado de acuerdo con la ley reguladora de investigación biomédica de Costa Rica y adjuntar la carta de aprobación del CEC cuando se envía el manuscrito. Manuscritos que reporten experimentos con animales deben incluir una nota que asegure que se cumplieron las recomendaciones generales para el manejo y cuidado de animales de experimentación. Se deben identificar con precisión los medicamentos y químicos utilizados en el estudio, incluyendo nombre genérico, dosis y vía de administración; además, deben describirse claramente los análisis estadísticos y el software utilizado, y emplear unidades de medida convencional aprobadas (UI o SI). AMC se reserva el derecho de solicitar el protocolo utilizado en la investigación para aclarar algún punto del trabajo.

Resultados. Presentar los resultados de forma clara y concisa, en secuencia lógica en el texto, cuadros, figuras e ilustraciones. No se repiten en el texto datos presentados en los cuadros o ilustraciones, ni se comenta ningún resultado en esta sección. La sección no puede estar compuesta sólo por figuras y cuadros.

Discusión. No repite información ofrecida en otra sección (introducción, resultados o métodos); menciona los aspectos nuevos e importantes 